\title{
Synthesis 2-Bromo-6-(4,5-Diphenyl-1-((1-Phenyl-1H- 1,2,3-Triazol-4-yl) Methyl)-1H-Imidazol-2-yl) Pyridine Derivatives: Evaluation of Anticancer Activity and Molecular Docking Studies
}

\author{
Ramdas Lakavath \\ Osmania University \\ Veeranna Dharmasothu \\ Osmania University \\ Ravi Guguloth \\ Osmania University \\ Sushmitha Bujji \\ Osmania University \\ Jadhav Ramchader ( $\square$ ramorgchemou@gmail.com ) \\ Osmania University
}

\section{Research Article}

Keywords: 1,2,3- triazole-pyridine hybrids, anticancer activity and molecular docking study

Posted Date: February 14th, 2022

DOI: https://doi.org/10.21203/rs.3.rs-1334374/v1

License: (c) (i) This work is licensed under a Creative Commons Attribution 4.0 International License.

Read Full License 


\section{Abstract}

A series of novel 2-bromo-6-(4,5-diphenyl-1-((subtituted-phenyl-1 H-1,2,3-triazol-4-yl)methyl)-1 H-imidazol-2yl)pyridine (7a-l) were synthesized and characterized by various analytical techniques. All the synthesized compounds were evaluated for their in vitro anticancer activity against two human cancer cell lines such as HT-1080 (Human fibro sarcoma cells) and Caco-2(Human colorectal adenocarcinoma cells) and $\mathrm{IC}_{50}$ values were compared against standard anticancer drug doxorubicin. Among all the synthesized compounds $7 \mathrm{~b}, 7 \mathrm{c}$ and $\mathbf{7 f}$ has shown potent activity against HT-1080. Similarly compounds $7 \mathrm{~b}$ and $7 \mathrm{c}$ shown significant activity among all the synthesized compounds against Caco-2. Furthermore, the results were supported by molecular docking studies.

\section{Introduction}

Cancer has become one of the major global health concerns Since it is the second leading cause of death after cardiovascular diseases, it remains a major health problem around the world ${ }^{1}$. Cancer is a collection of related diseases that involve abnormal cell growth with the potential to invade or spread to other parts of the body. Since it involves the conversion of any normal cells to a cancerous cell showing tandem replication and cell divisions at much faster rate in comparison to the normal cells and thus provides a potential target for the development of chemotherapeutic agents. The worldwide occurred estimated deaths and new cancer cases in 2020 were 10.0 million and 19.3 million. The cancer burden globally is expected to be raise $47 \%$ from 2020 to 2040 with 28.4 million cases ${ }^{2}$. Chemo resistance is an important phenomenon associated with the cell division. In addition, safety profile and side effects are the major concerns with anticancer drugs. Therefore, the development of novel agents with increased efficacy while reducing the side effects will encourage the researchers towards the drug design and development.

1,2,3-Triazoles, are the most important nitrogen containing heterocycles, and these derivatives possess diverse biological properties Moreover, some of 1,2,3-triazole containing anticancer agents such as Cefatrizine[a] and Carboxyamido-triazole[b] (Fig. 1) have already been under clinical evaluation for the treatment of cancer.

Derivatives of Pyridine are the privileged scaffolds with wide range of pharmacological properties, which plays an important role by inhibition of CDK, EGFR, PI3K, and RGGT etc in different cancers ${ }^{3,4}$. Anti-cancer agents like Masitinib and ABT-751 (E7010) containing pyridine as a core moiety have already been under clinical trials for the treatment of cancers, indicating the importance of pyridine derivatives in the discovery of anticancer agents ${ }^{5}$.All 1,2,3-triazole-pyridine hybrids Fig-2 with $\mathrm{IC}_{50}$ : 0.1-19.5 mM, showed considerable activity against HT-29, DU-145 and A549 cancer cell lines, and most of them were not less active than ABT-751 IC50: 1.31-1.62 $\mathrm{mM}$ and Nocodazole with $\mathrm{IC}_{50}: 2.2-3.1 \mathrm{mM}^{6,7}$.

A series of 1,2,3- triazole-pyridine hybrids Fig-3 was highly active against the two enzymes NAMPT and HDAC with $\mathrm{IC}_{50}$ value $18-190 \mathrm{nM}^{8,9}$. 
Hybrid molecules have the potential to reduce side effects and overcome the drug resistance since hybrids with two or more different pharmacophores may also own multiple action mechanism

Considering the anti-cancer activity exhibited by both 1,2,3- triazole derivatives and the pyridine derivatives from literature search and that fusion of 1,2,3- triazole and pyridine could result in molecules having greater anti-cancer activity due to synergistic effect of both 1,2,3-triazole-pyridine scaffolds. Hence, a series of 1,2,3- triazole-pyridine hybrids have been synthesized and evaluated for their in vitro anti-cancer activity using human cell lines with results supported by docking studies.

\section{Results And Discussion \\ Chemistry}

Synthetic route for the 2-bromo-6-(4,5-diphenyl-1-((subtituted-phenyl-1 $H+1,2,3$-triazol-4-yl) methyl)-1Himidazol-2-yl) pyridine (7a-l) were summarized in scheme 1. The 1,2,3-triazole tethered pyridine core nucleus was constructed from commercially available starting materials. The synthesis was carried as one pot three component condensation of 6-bromopicolinaldehyde (1) benzil (2) and ammonium acetate in presence of ethanol in acetic acid at $70^{\circ} \mathrm{C}$ for $4 \mathrm{hrs}$ to afford compound(3)with the propargylation of using propargyl bromide (4) dry DMF and dry $\mathrm{K}_{2} \mathrm{CO}_{3}$ it for 3-4hrs to yield2-bromo-6-(4,5-diphenyl-1-(prop2-yn-1-yl)-1 H-imidazol-2-yl)pyridine (5)which on further click reaction of substituted aryl azides (6a-l) at the terminal alkyne position to obtain2-bromo-6-(4,5-diphenyl-1-((subtituted-phenyl-1 H-1,2,3-triazol-4yl)methyl)-1 $H$-imidazol-2-yl)pyridinepure compounds (7a-l) in the range of yields (70-80\%).

\section{Biological Evaluation and Structural Activity Relationship (SAR):}

The newly synthesized compounds (7a-l) were screened for their in vitro anti-cancer activity using two human cancer cell lines such as HT-1080 (Human fibro sarcoma cells) and Caco-2 (Human colorectal adenocarcinoma cells) by MTT assay. Doxorubicin was used as a reference anti-cancer drug and the results were expressed in terms of $\mathrm{IC}_{50}(\mu \mathrm{M})$ values as summarized in Table 1.

Among the synthesized compounds, 7b, and 7c shown more potent anti-cancer activity against HT-1080 and Caco-2 cell lines, compared to control drug. It showed that compound (7b) with 4-Bromo group on the phenyl ring exhibited more potent activity than the control doxorubicin. Similarly substitution with strong electron withdrawing agent like chloro group at ortho postion on the phenyl ring resulted compound 7c shown slightly decrease in activity compared to $\mathbf{7 b}$. whereas Replacement of 4-bromo on the phenyl ring with 4-chloro Resulted compound 7d which showed lower activity than $\mathbf{7 b}$. The unsubstituted and alkyl substitutions on the phenyl ring were not tolerated. Compound $\mathbf{7 g}$ with electron donating agent, 4methoxy substitution shown slightly decrease in activity compared to control drug. Shifting of methoxy substitution from ortho position to para position compound resulted in loss of activity. 
Table 1

The $\mathrm{IC}_{50}$ values of compounds $7 \mathrm{a}-\mathrm{I}$

\begin{tabular}{|lll|}
\hline Compound Name & IC $_{50}$ in $\mu \mathrm{M}$ at 72 hr's & \\
\hline & HT-1080 & Caco-2 \\
& (Human fibro sarcoma cells) & (Human colorectal adenocarcinoma cells) \\
\hline $7 \mathrm{a}$ & $47.5 \pm 1.28$ & $41.62 \pm 1.06$ \\
\hline $7 \mathrm{~b}$ & $9.5 \pm 1.64$ & $12.29 \pm 1.21$ \\
\hline $7 \mathrm{c}$ & $10.8 \pm 2.46$ & $24.53 \pm 1.48$ \\
\hline $7 \mathrm{~d}$ & $36.2 \pm 1.92$ & $53.11 \pm 1.76$ \\
\hline $7 \mathrm{e}$ & $43.4 \pm 1.47$ & $58.12 \pm 1.21$ \\
\hline $7 \mathrm{f}$ & $12.4 \pm 2.29$ & $32.62 \pm 1.18$ \\
\hline $7 \mathrm{~g}$ & $42.4 \pm 1.34$ & $47.15 \pm 1.23$ \\
\hline $7 \mathrm{~h}$ & $29.4 \pm 2.02$ & $39.30 \pm 1.82$ \\
\hline $7 \mathrm{i}$ & $30.8 \pm 1.69$ & $46.31 \pm 1.01$ \\
\hline $7 \mathrm{j}$ & $47.6 \pm 2.13$ & $52.79 \pm 1.35$ \\
\hline $7 \mathrm{k}$ & $43.1 \pm 1.29$ & $49.73 \pm 1.28$ \\
\hline $7 \mathrm{I}$ & $33.8 \pm 1.95$ & $46.71 \pm 1.53$ \\
\hline Doxorubicin & $11.2 \pm 1.04$ & $6.17 \pm 1.88$ \\
\hline
\end{tabular}

\section{Molecular docking studies:}

The ligands were sketched in chemdraw and saved it in mol2 format. All the sketched molecules were converted to energy minimized 3D structures by using ligprep module for in-silico protein - ligand docking using Schrödinger 11.4. Each molecule was docked separately. Initially the molecule was loaded; torsions were set and saved it in PDB format. All the heteroatoms were removed from the 5FGK. PDB (CDK8-CYCC IN COMPLEX WITH 8-[3-(3-Amino-1H-indazol-6-yl)-5-chloro- pyridine-4-yl]-2,8-diaza-spiro [4.5] decan-1-one). The Mediator complex-associated cyclin-dependent kinase CDK8 has been implicated in human disease, particularly in colorectal cancer where it has been reported as a putative oncogene to make complex receptor free of any ligand before docking. Receptor grid generated using glide module. The best conformation was chosen with the lowest docked energy after the docking search was completed. The interactions of 5FGK protein and ligand conformations, including hydrogen bonds and the bond lengths were analyzed. Molecular docking study was performed by using maestro (Schrödinger 11.4) which was a suite of automated docking tools and was used to predict the affinity, activity, binding orientation of ligand with the target protein and to analyze best conformations, the protein with all the 
compounds (7a-l) were loaded individually evaluated. We observed that in the compound $7 \mathrm{~b}$ molecule two aromatic rings showing $\Pi-\Pi$ stacking interactions with aminoacids histidine and tryptophan, Triazole \& Imidazole rings showing cation pi interaction with amino acid arginine, pyridine moiety showing П-П stacking interactions with aminoacid Tyrosineand showed best fit, potent dock score then compared with doxorubicin.

Table 2

The Dock scores of synthesized molecules

\begin{tabular}{|ll|}
\hline Compound name & Docking score \\
\hline $5 F G K$ & \\
\hline $7 a$ & -6.183 \\
\hline $7 b$ & -6.783 \\
\hline $7 c$ & -5.98 \\
\hline $7 d$ & -5.156 \\
\hline $7 e$ & -5.87 \\
\hline $7 f$ & -6.185 \\
\hline $7 g$ & -5.517 \\
\hline $7 \mathrm{~h}$ & -5.982 \\
\hline $7 \mathrm{i}$ & -5.522 \\
\hline $7 \mathrm{~J}$ & -5.749 \\
\hline $7 \mathrm{k}$ & -5.256 \\
\hline $7 \mathrm{l}$ & -5.277 \\
\hline Doxorubicin & -7.065 \\
\hline
\end{tabular}

\section{Conclusion}

In conclusion, a series of 1,2,3-triazole linked pyridine hybrids derivatives (7a-l) were synthesized and characterized by ${ }^{1} \mathrm{HNMR},{ }^{13} \mathrm{CNMR}$, and mass spectral analysis. Further, these synthesized compounds were tested for their anticancer activity against two human cancer cell lines, HT-1080(Human fibro sarcoma cells), Caco-2 (Human colorectal adenocarcinoma cells). Doxorubicin is used as a control. 
Among them, compounds $\mathbf{7 b}, \mathbf{7 c}$ and $\mathbf{7 f}$ showed potent anticancer activity compared to control drug. Docking results shown that compounds exhibited potent dock score then compared with doxorubicin.

\section{Experimental}

\section{General experimental methods:}

Were purchased all the chemical of the organic reagents and solvents from Tci, Merk, were used without further. ${ }^{1} \mathrm{H}$ NMR and ${ }^{13} \mathrm{C}$ NMR spectra were determined in DMSO by using 500 and $125 \mathrm{MHz}$ spectrometers (Instrument Bruker Avance II $500 \mathrm{MHz}$ ). Chemical shift values are displayed as ppm and spin multiplicities are indicated as singlet (s); doublet (d); doublet of doublet (dd); triplet(t); multiplets (m); and coupling constants are shown in hertz. Column chromatography was performed on silica gel (60-120 mesh) using distilled hexane and ethyl acetate solvents. Mass and Infrared spectra were recorded on QSTAR XL GCMS, Shimadzu FT-IR-8400s mass spectrometer. Melting points were determined in open glass capillary tube on a DbkProg. Melting Point apparatus and were uncorrected.

\section{General procedure for the preparation of 2-bromo-6-(4,5-diphenyl-1 H -imidazol-2-yl) pyridine (3)}

The synthetic route for the 2-bromo-6-(4,5-diphenyl-1 H-imidazol-2-yl) pyridine (3) was carried out by one pot three component condensation of 6-bromopicolinaldehyde (1), benzil (2) and ammonium acetate and catalytical amount of acetic acid in ethanol at $70^{\circ} \mathrm{C}$ for $5 \mathrm{hrs}$ to afford compound (3)

General procedure for the preparation of 2-bromo-6-(4,5-diphenyl-1-(prop-2-yn-1-yl)-1 H -imidazol-2-yl) pyridine (5)

The synthetic route for the 2-bromo-6-(4,5-diphenyl-1-(prop-2-yn-1-yl)-1 H-imidazol-2-yl) pyridine (5) was carried out by proporgylation of compound (3) with propergylic bromide (4) dry DMF and dry $\mathrm{K}_{2} \mathrm{CO}_{3}$ at it for $4 \mathrm{hrs}$ proporgylation at the position of free $\mathrm{N}-\mathrm{H}$ group yields to -propargylated compound bromo-6-(4,5diphenyl-1-(prop-2-yn-1-yl)-1 H-imidazol-2-yl) pyridine (5)

General procedure for the preparation of 2-bromo-6-(4,5-diphenyl-1-((substituted-phenyl-1H-1,2,3-triazol-4yl) methyl)-1 H -imidazol-2-yl) pyridine (7a-l)

Synthesis of 2-bromo-6-(4,5-diphenyl-1-((substituted-phenyl-1H-1,2,3-triazol-4-yl) methyl)-1 H-imidazol-2-yl) pyridine (7a-l) $(7 a-1)$ were carried out by click reaction of propargylated compound (5) $(0.1 \mathrm{mmol})$ with different aryl azides (6a-l) $(0.2 \mathrm{mmol})$ using Click chemistry in $\mathrm{CuSO}_{4} \cdot 5 \mathrm{H}_{2} \mathrm{O}$ with sodium ascorbate and DMF at room temperature for 8 hours. The completion of the reaction was monitored by TLC. Upon completion of the reaction mass were purified by column chromatography using hexane/ ethyl acetate (2:3 v/v) to afford 2-bromo-6-(4,5-diphenyl-1-((substituted-phenyl-1 H-1,2,3-triazol-4-yl) methyl)-1 Himidazol-2-yl) pyridine (7a-l) gave excellent yields $70-80 \%$.

\section{MTT Assay Principle:}


This MTT assay is a colorimetric assay that measures the reduction of 3-(4,5-dimethylthiazol- 2-yl)-2,5diphenyl tetrazolium bromide (MTT) by mitochondrial succinate dehydrogenase. The MTT enters the cells and passes into the mitochondria where it gets reduced and forms insoluble dark purple formazan crystals. The crystals are then solubilized with an organic solvent DMSO and the soluble formazan product is measured by reading absorbance at $570 \mathrm{~nm}$ with spectrophotometer. Since reduction of MTT can only occur in metabolically active cells, the level of activity is the measure of the viability of the cells. The HT-1080 \& Caco-2 cellswere seeded in 96-well plates with density of $10^{5}$ cells per well in $100 \mu \mathrm{L}$ of complete medium and allowed to grow overnight for attachment. The cells are then treated with various concentrations $(5,10,20,40,60,80$ and $100 \mu \mathrm{M})$ of test samples C8 and C10 in triplicate for $24 \mathrm{hr}$ and incubated for $72 \mathrm{hr}$. After treatment, the cells were washed twice with PBS. Then $15 \mu \mathrm{L}$ MTT reagent in PBS medium with concentration of $0.5 \mathrm{mg} / \mathrm{mL}$ was added each well and adjusted the volume then the cells were incubated for 3 hours at $37^{\circ} \mathrm{C}$ until intracellular purple formazan crystals are visible under microscope. Then MTT reagent was aspirated and the formazan crystals formed were dissolved by the addition of $100 \mu \mathrm{L}$ of DMSO for $1 \mathrm{hr}$ at $37^{\circ} \mathrm{C}$. The quantity of formazan was measured by using a absorbance plate reader at $570 \mathrm{~nm}$ wavelength.

\section{Spectral Data}

2-bromo-6-(4,5-diphenyl-1-((1-phenyl-1 H1,2,3-triazol-4-yl)methyl)-1 Himidazol-2-yl)pyridine (7a) Yield 72\%, mp: 139-141 ${ }^{\circ} \mathrm{C} ; \mathrm{Rf}=0.30$ (EtOAc:n-Hexane 2:3); ${ }^{1} \mathrm{H}$ NMR NMR (500 MHz, DMSO-d6) $\delta 8.49$ (s, 1H), 7.97 $(d, J=7.85 \mathrm{~Hz}, 1 \mathrm{H}), 7.77(\mathrm{~d}, \mathrm{~J}=7.57 \mathrm{~Hz}, 2 \mathrm{H}), 7.71(\mathrm{~d}, \mathrm{~J}=7.85 \mathrm{~Hz}, 2 \mathrm{H}), 7.63(\mathrm{t}, \mathrm{J}=7.85 \mathrm{~Hz}, 1 \mathrm{H}), 7.57(\mathrm{~d}, \mathrm{~J}$ $=7.85 \mathrm{~Hz}, 2 \mathrm{H}), 7.43(\mathrm{dd}, \mathrm{J}=7.85,7.45 \mathrm{~Hz}, 4 \mathrm{H}), 7.41(\mathrm{t}, \mathrm{J}=7.45 \mathrm{~Hz}, 1 \mathrm{H}), 7.40(\mathrm{dd}, \mathrm{J}=7.57,7.20 \mathrm{~Hz}, 2 \mathrm{H})$, $7.40(\mathrm{~d}, \mathrm{~J}=7.85 \mathrm{~Hz}, 1 \mathrm{H}), 7.35(\mathrm{t}, \mathrm{J}=7.20 \mathrm{~Hz}, 1 \mathrm{H}), 7.33(\mathrm{t}, \mathrm{J}=7.45 \mathrm{~Hz}, 1 \mathrm{H}), 5.33(\mathrm{~s}, 2 \mathrm{H}) \cdot{ }^{3} \mathrm{C} \mathrm{NMR}(125 \mathrm{MHz}$, DMSO-d6) $\delta 148.7,145.1,144.5,139.2,138.5,135.2,134.7,133.6,130.1,129.4,128.9,128.4,128.3,128.2$, 127.8, 127.6, 126.8, 125.8, 123.6, 120.7, 47.3.LC-MS m/z: 535 [M+H]+ Elemental analysis, Calculated, $\%: \mathrm{C}_{29} \mathrm{H}_{21} \mathrm{BrN}_{6}: \mathrm{C}, 65.30 ; \mathrm{H}, 3.97 ; \mathrm{N}, 15.75$; Found \%:C, 65.25; H, 3.89; N, 15.69;

\section{2-bromo-6-(1-((1-(4-bromophenyl)-1 H1,2,3-triazol-4-yl)methyl)-4,5-diphenyl-1 Himidazol-2-yl)pyridine}

(7b)Yield 78\%, mp: 143-145 C; Rf = 0.42 (EtOAc:n-Hexane 2:3); ${ }^{1} \mathrm{H}$ NMR (500 MHz, DMSO-d6) $\delta 8.48$ (s, 1H), $7.92(d, J=7.85 \mathrm{~Hz}, 1 \mathrm{H}), 7.81(\mathrm{~d}, \mathrm{~J}=8.30 \mathrm{~Hz}, 2 \mathrm{H}), 7.74(\mathrm{~d}, \mathrm{~J}=7.85 \mathrm{~Hz}, 2 \mathrm{H}), 7.64$ (dd, J = 7.85, 7.48 $\mathrm{Hz}, 1 \mathrm{H}), 7.57(\mathrm{~d}, \mathrm{~J}=7.85 \mathrm{~Hz}, 2 \mathrm{H}), 7.53(\mathrm{~d}, \mathrm{~J}=8.30,9.30 \mathrm{~Hz}, 2 \mathrm{H}), 7.43(\mathrm{dd}, \mathrm{J}=7.85,7.45 \mathrm{~Hz}, 2 \mathrm{H}), 7.41$ (d, J $=7.85 \mathrm{~Hz} 1 \mathrm{H}), 7.40(\mathrm{t}, \mathrm{J}=7.45 \mathrm{~Hz}, 1 \mathrm{H}), 7.40(\mathrm{dd}, \mathrm{J}=7.85,7.45 \mathrm{~Hz}, 2 \mathrm{H}), 7.40(\mathrm{dd}, \mathrm{J}=7.85,7.45 \mathrm{~Hz}, 1 \mathrm{H})$, $7.34(\mathrm{t}, \mathrm{J}=7.45 \mathrm{~Hz}, 1 \mathrm{H}), 5.35(\mathrm{~s}, 2 \mathrm{H}) ;{ }^{13} \mathrm{C}$ NMR $(125 \mathrm{MHz}$, DMSO-d 6 ) $\delta 145.21,142.13,140.14,139.72$, 135.77, 133.68, 133.41, 132.14, 130.27, 128.73, 128.37, 127.83, 127.67, 127.30, 127.04, 125.07, 124.33, $124.06,122.03,121.02,120.12,119.54,118.95,118.82,110.41,109.67,40.13,38.12 . L C-M S \mathrm{~m} / \mathrm{z}:$ $613[\mathrm{M}+\mathrm{H}]+$ Elemental analysis, Calculated, \%: $\mathrm{C}_{29} \mathrm{H}_{20} \mathrm{Br}_{2} \mathrm{~N}_{6}: \mathrm{C}, 56.88 ; \mathrm{H}, 3.29 ; \mathrm{N}, 13.72 ;$ Found \%:C, 56.81; H, 3.21; N, 13.65;

2-bromo-6-(1-((1-(2-chlorophenyl)-1 H1,2,3-triazol-4-yl)methyl)-4,5-diphenyl-1 Himidazol-2-yl)pyridine (7c)Yield 73\%, mp: 151-153 º; Rf = 0.38 (EtOAc:n-Hexane 2:3); ${ }^{1} \mathrm{H}$ NMR (500 MHz, DMSO-d6) $\delta 8.55$ (s, 
1H), $8.10(\mathrm{dd}, \mathrm{J}=8.01,1.03 \mathrm{~Hz}, 1 \mathrm{H}), 7.79(\mathrm{dd}, \mathrm{J}=7.51,2.02 \mathrm{~Hz}, 1 \mathrm{H}), 7.74(\mathrm{~m}, 2 \mathrm{H}), 7.66(\mathrm{t}, \mathrm{J}=8.01 \mathrm{~Hz}, 1 \mathrm{H})$, $7.56(\mathrm{~m}, 2 \mathrm{H}), 7.46-7.36(\mathrm{~m}, 10 \mathrm{H}), 5.26(\mathrm{~s}, 2 \mathrm{H}) ;{ }^{13} \mathrm{C}$ NMR $\left(125 \mathrm{MHz}\right.$, DMSO $\left.\mathrm{d}_{6}\right) \delta$ 145.43, 141.87, 140.26, $139.94,135.84,133.96,133.84,132.02,131.96,130.27,128.76,128.52,128.30,127.98,127.83,127.60$, $127.51,127.49,127.03,125.09,124.38,124.17,122.05,121.37,120.50,119.38,118.94,110.42,109.36$, 40.36, 38.52.LC-MS m/z: $568[\mathrm{M}+\mathrm{H}]+$ Elemental analysis, Calculated, \%: $\mathrm{C}_{29} \mathrm{H}_{20} \mathrm{BrClN}_{6}: \mathrm{C}, 61.34 ; \mathrm{H}, 3.55$; $\mathrm{N}, 14.80$; Found \%:C, 61.27; H, 3.48; N, 14.71;

\section{2-bromo-6-(1-((1-(4-chlorophenyl)-1 H1,2,3-triazol-4-yl)methyl)-4,5-diphenyl-1 Himidazol-2-yl)pyridine}

(7d)Yield 75\%, mp: 157-159 ' C; Rf = 0.35 (EtOAc:n-Hexane 2:3); ${ }^{1} \mathrm{H}$ NMR (500 MHz, DMSO-d6) $\delta 8.49$ (s, $1 \mathrm{H}), 7.92(\mathrm{~d}, \mathrm{~J}=7.85 \mathrm{~Hz}, 1 \mathrm{H}), 7.74(\mathrm{~d}, \mathrm{~J}=7.85 \mathrm{~Hz}, 2 \mathrm{H}), 7.73(\mathrm{~d}, \mathrm{~J}=8.43 \mathrm{~Hz}, 2 \mathrm{H}), 7.63$ (dd, J = 7.85, 7.44 $\mathrm{Hz}, 1 \mathrm{H}), 7.59(\mathrm{~d}, \mathrm{~J}=7.85 \mathrm{~Hz}, 2 \mathrm{H}), 7.48(\mathrm{~d}, \mathrm{~J}=8.43 \mathrm{~Hz}, 2 \mathrm{H}), 7.43(\mathrm{dd}, \mathrm{J}=7.85,7.45 \mathrm{~Hz}, 2 \mathrm{H}), 7.41(\mathrm{~d}, \mathrm{~J}=$ $7.85 \mathrm{~Hz}, 1 \mathrm{H}), 7.40(\mathrm{~m}, 3 \mathrm{H}), 7.34(\mathrm{t}, \mathrm{J}=7.45 \mathrm{~Hz}, 1 \mathrm{H}), 5.36(\mathrm{~s}, 2 \mathrm{H}) ;{ }^{13} \mathrm{C}$ NMR $(125 \mathrm{MHz}$, DMSO-d 6 ) $\delta 145.48$, $142.13,140.26,139.75,135.82,133.82,132.97,132.53,132.08,130.34,130.26,128.74,158.35,127.86$, $127.64,127.49,127.06,125.07,124.39,124.18,122.07,121.47,119.56,118.94,110.42,109.32,40.12$, 38.17.LC-MS m/z: 568[M+H]+ Elemental analysis, Calculated, \%: $\mathrm{C}_{29} \mathrm{H}_{20} \mathrm{BrClN}_{6}: \mathrm{C}, 61.34 ; \mathrm{H}, 3.55 ; \mathrm{N}$, 14.80 ; Found \%:C, 61.27; H, 3.48; N, 14.71;

4-(4-((2-(6-bromopyridin-2-yl)-4,5-diphenyl-1 Himidazol-1-yl)methyl)-1 H-1,2,3-triazol-1-yl)phenol (7e)Yield 70\%, mp: $135-137{ }^{\circ} \mathrm{C} ; \mathrm{Rf}=0.28$ (EtOAc:n-Hexane 2:3); ${ }^{1} \mathrm{H}$ NMR (500 MHz, DMSO-d6) $\delta 9.57$ (s, 1H) 8.60 $(\mathrm{s}, 1 \mathrm{H}), 7.97(\mathrm{~d}, \mathrm{~J}=7.85 \mathrm{~Hz}, 1 \mathrm{H}), 7.71(\mathrm{~d}, \mathrm{~J}=7.85 \mathrm{~Hz}, 2 \mathrm{H}), 7.62(\mathrm{t}, \mathrm{J}=7.85 \mathrm{~Hz}, 1 \mathrm{H}), 7.59(\mathrm{~d}, \mathrm{~J}=8.43 \mathrm{~Hz}$, 2H), 7.57 (d, J = 7.85 Hz, 2H), 7.43 (dd, J = 7.85, 7.45 Hz, 2H), 7.41 (dd, J = 7.85, 7.45 Hz, 2H), 7.40 (t, J = $7.45 \mathrm{~Hz}, 1 \mathrm{H}), 7.40(\mathrm{~d}, \mathrm{~J}=7.85 \mathrm{~Hz}, 1 \mathrm{H}), 7.33(\mathrm{t}, \mathrm{J}=7.45 \mathrm{~Hz}, 1 \mathrm{H}), 6.76(\mathrm{~d}, \mathrm{~J}=8.43 \mathrm{~Hz}, 2 \mathrm{H}), 5.34(\mathrm{~s}, 2 \mathrm{H}) .{ }^{13} \mathrm{C}$ NMR $(125$ MHz, DMSO-d6) $\delta 157.3,148.7,145.5,144.5,140.6,139.2,138.5,135.2,133.6,130.1,128.9$, 128.4, 128.2, 127.8, 127.6, 125.8, 124.1, 120.7, 119.1, 115.2, 47.3. LC-MS m/z: 550[M+H]+ Elemental analysis, Calculated, \%: $\mathrm{C}_{29} \mathrm{H}_{21} \mathrm{BrN}_{6} \mathrm{O}: \mathrm{C}, 63.40 ; \mathrm{H}, 3.85 ; \mathrm{N}, 15.30$; Found \%:C, 63.32; $\mathrm{H}, 3.79 ; \mathrm{N}, 15.21$;

\section{2-bromo-6-(1-((1-(2-methoxyphenyl)-1 H-1,2,3-triazol-4-yl)methyl)-4,5-diphenyl-1 Himidazol-2-yl)pyridine}

(7f)Yield 72\%, mp: $129-131{ }^{\circ} \mathrm{C} ; \mathrm{Rf}=0.40$ (EtOAc:n-Hexane 2:3); ${ }^{1} \mathrm{H}$ NMR(500 MHz, DMSO-d6) $\delta 8.48$ (s, 1H) $7.90(\mathrm{~d}, J=7.85 \mathrm{~Hz}, 1 \mathrm{H}), 7.71(\mathrm{~d}, J=7.85 \mathrm{~Hz}, 2 \mathrm{H}), 7.63(\mathrm{dd}, \mathrm{J}=7.85,7.47 \mathrm{~Hz}, 1 \mathrm{H}), 7.59$ (d, J = 7.85 $\mathrm{Hz}, 2 \mathrm{H}), 7.49$ (d, J = 7.85 Hz, 1H), $7.43(\mathrm{dd}, \mathrm{J}=7.85,7.45 \mathrm{~Hz}, 2 \mathrm{H}), 7.41(\mathrm{~d}, \mathrm{~J}=7.85 \mathrm{~Hz}, 1 \mathrm{H}), 7.40(\mathrm{~m}, \mathrm{~J}=$ 7.45, 7.85 Hz, 3H), $7.34(\mathrm{t}, \mathrm{J}=7.45 \mathrm{~Hz}, 1 \mathrm{H}), 7.26(\mathrm{dd}, \mathrm{J}=7.857 .44 \mathrm{~Hz}, 1 \mathrm{H}), 7.06(\mathrm{~d}, \mathrm{~J}=7.85 \mathrm{~Hz}, 1 \mathrm{H}), 6.97$ (dd, J = 7.85 7.44 Hz, 1H), 5.35 (s, 2H), 3.82 (s, 3H); ${ }^{13} \mathrm{C}$ NMR (125 MHz, DMSO-d 6 ) ठ 154.60, 145.42, $141.53,140.39,139.68,135.82$, 133.85, 132.06, 130.27, 128.74, 128.46, 128.37, 127.85, 127.59, 127.02, $125.06,124.37,124.23,123.09,122.04,120.84,118.95,118.32,114.72,110.48,109.36,55.69,40.32$, 38.52.LC-MS m/z: $564[\mathrm{M}+\mathrm{H}]+$ Elemental analysis, Calculated, \%: $\mathrm{C}_{30} \mathrm{H}_{23} \mathrm{BrN}_{6} \mathrm{O}: \mathrm{C}, 63.95 ; \mathrm{H}, 4.11 ; \mathrm{N}, 14.92$; Found \%:C,63.87; $\mathrm{H}, 4.01 ; \mathrm{N}, 14.84$;

2-bromo-6-(1-((1-(4-methoxyphenyl)-1 H1,2,3-triazol-4-yl)methyl)-4,5-diphenyl-1 Himidazol-2yl)pyridine(7g) Yield $74 \%, \mathrm{mp:} 125-127^{\circ} \mathrm{C} ; \mathrm{Rf}=0.38$ (EtOAc:n-Hexane 2:3); 
${ }^{1} \mathrm{H}$ NMR(500 MHz, DMSO-d6) $\delta 8.49(\mathrm{~s}, 1 \mathrm{H}) 7.92(\mathrm{~d}, \mathrm{~J}=7.85 \mathrm{~Hz}, 1 \mathrm{H}), 7.73(\mathrm{~d}, \mathrm{~J}=7.85 \mathrm{~Hz}, 2 \mathrm{H}), 7.64$ (dd, J $=7.85,7.47 \mathrm{~Hz}, 1 \mathrm{H}), 7.61(\mathrm{~d}, \mathrm{~J}=7.85 \mathrm{~Hz}, 2 \mathrm{H}), 7.51(\mathrm{~d}, \mathrm{~J}=7.85 \mathrm{~Hz}, 1 \mathrm{H}), 7.43(\mathrm{dd}, \mathrm{J}=7.85,7.45 \mathrm{~Hz}, 2 \mathrm{H})$, $7.43(\mathrm{~d}, \mathrm{~J}=7.85 \mathrm{~Hz}, 1 \mathrm{H}), 7.42(\mathrm{~m}, \mathrm{~J}=7.45,7.85 \mathrm{~Hz}, 3 \mathrm{H}), 7.30$ (d, J = $7.857 .44 \mathrm{~Hz}, 2 \mathrm{H}), 7.09$ (d, J = 7.85

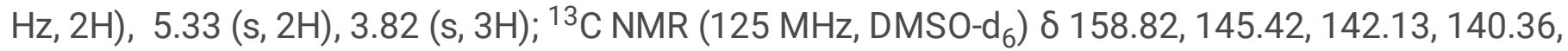
$139.72,135.82,133.87,132.06,130.28,128.75,128.36,128.29,127.84,127.53,127.03,125.03,124.35$, 124.29, 122.07, 120.98, 119.53, 118.97, 118.72, 116.23, 110.72, 109.38, 55.87, 40.17, 38.12.LC-MS m/z: $564[\mathrm{M}+\mathrm{H}]+$ Elemental analysis, Calculated, \%: $\mathrm{C}_{30} \mathrm{H}_{23} \mathrm{BrN}_{6} \mathrm{O}: \mathrm{C}, 63.95 ; \mathrm{H}, 4.11 ; \mathrm{N}, 14.92$; Found \%:C,63.87; $\mathrm{H}, 4.01 ; \mathrm{N}, 14.84$;

2-bromo-6-(4,5-diphenyl-1-((1-(o-tolyl)-1 H-1,2,3-triazol-4-yl)methyl)-1 Himidazol-2-yl)pyridine (7h)Yield 73\%, mp: 119-121 ${ }^{\circ} \mathrm{C} ; \mathrm{Rf}=0.42$ (EtOAc:n-Hexane 2:3); ${ }^{1} \mathrm{H}$ NMR (500 MHz, DMSO-d6) $\delta 8.50$ (s, 1H), 8.10 $(\mathrm{dd}, \mathrm{J}=8.02,1.03 \mathrm{~Hz}, 1 \mathrm{H}), 7.72(\mathrm{~m}, 2 \mathrm{H}), 7.66(\mathrm{t}, \mathrm{J}=8.01 \mathrm{~Hz}, 1 \mathrm{H}), 7.56(\mathrm{~m}, 2 \mathrm{H}), 7.51-7.27(\mathrm{~m}, 11 \mathrm{H}), 5.26$ (s, 2H), 2.27 (s, 3H); ${ }^{13} \mathrm{C}$ NMR (125 MHz, DMSO-d 6 ) ठ 145.47, 141.56, 140.13, 139.05, 135.82, 135.34, $133.85,132.06,130.95,130.24,129.26,128.74,128.36,127.98,127.63,127.54,127.31,127.25,127.09$, $125.08,124.35,124.26,122.05,120.37,119.63,118.95,118.54,110.47,109.38,40.32$, 38.53, 17.39.LCMS m/z: $548[\mathrm{M}+\mathrm{H}]+$ Elemental analysis, Calculated, \%: $\mathrm{C}_{30} \mathrm{H}_{23} \mathrm{BrN}_{6}: \mathrm{C}, 65.82 ; \mathrm{H}, 4.23 ; \mathrm{N}, 15.35 ;$ Found $\%: C, 65.73 ; H, 4.17 ; \mathrm{N}, 15.28$;

2-bromo-6-(4,5-diphenyl-1-((1-(p-tolyl)-1 H1,2,3-triazol-4-yl)methyl)-1 Himidazol-2-yl)pyridine(7i)Yield 77\%, $\mathrm{mp}: 123-125^{\circ} \mathrm{C} ; \mathrm{Rf}=0.45$ (EtOAc:n-Hexane 2:3); Found \%:C, 65.73; H, 4.17; N, 1528; ${ }^{1} \mathrm{H} \mathrm{NMR}(500 \mathrm{MHz}$, DMSO-d6) $\delta 8.54(\mathrm{~s}, 1 \mathrm{H}), 8.10(\mathrm{dd}, \mathrm{J}=8.02,1.03 \mathrm{~Hz}, 1 \mathrm{H}), 7.72(\mathrm{~m}, 2 \mathrm{H}), 7.66(\mathrm{t}, \mathrm{J}=8.01 \mathrm{~Hz}, 1 \mathrm{H}), 7.56(\mathrm{~m}$,

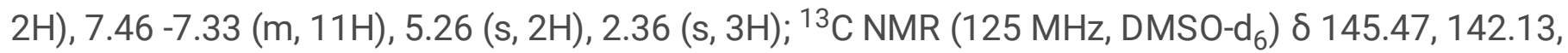
$140.18,139.69,137.85,135.89,133.84,132.09,131.95,130.82,130.25,128.74,128.39,127.76,127.63$, $127.59,127.28,125.06,124.36,124.28,122.04,119.68,119.35,119.08,118.94,110.47,109.38,40.18$, 38.17, 21.14.LC-MS m/z: $548[\mathrm{M}+\mathrm{H}]+$ Elemental analysis, Calculated, $\%: \mathrm{C}_{30} \mathrm{H}_{23} \mathrm{BrN}_{6}: \mathrm{C}, 65.82 ; \mathrm{H}, 4.23 ; \mathrm{N}$, 15.35;

1-(3-(4-((2-(6-bromopyridin-2-yl)-4,5-diphenyl-1 Himidazol-1-yl)methyl)-1 H1,2,3-triazol-1-yl)phenyl)ethan-1one(7j)Yield 75\%, mp: $143-145^{\circ} \mathrm{C} ; \mathrm{Rf}=0.37$ (EtOAc:n-Hexane 2:3);

${ }^{1} \mathrm{H}$ NMR (500 MHz, DMSO-d6) $\delta 8.56(\mathrm{~s}, 1 \mathrm{H}), 7.92(\mathrm{~d}, \mathrm{~J}=7.85 \mathrm{~Hz}, 1 \mathrm{H}), 7.90(\mathrm{~s}, 1 \mathrm{H}), 7.74(\mathrm{~d}, \mathrm{~J}=7.85 \mathrm{~Hz}$, 2H), $7.63(\mathrm{~d}, \mathrm{~J}=7.85 \mathrm{~Hz}, 2 \mathrm{H}), 7.59(\mathrm{~d}, \mathrm{~J}=7.85 \mathrm{~Hz}, 2 \mathrm{H}) 7.55(\mathrm{~d}, \mathrm{~J}=7.85 \mathrm{~Hz}, 1 \mathrm{H}), 7.48$ (dd, J = 7.87, 7.48 $\mathrm{Hz}, 1 \mathrm{H}) 7.43(\mathrm{dd}, \mathrm{J}=7.85,7.45 \mathrm{~Hz}, 2 \mathrm{H}), 7.41(\mathrm{~d}, \mathrm{~J}=7.85 \mathrm{~Hz}, 1 \mathrm{H}), 7.40(\mathrm{t}, \mathrm{J}=7.75 \mathrm{~Hz}, 3 \mathrm{H}), 7.34(\mathrm{t}, \mathrm{J}=7.45$ $\mathrm{Hz}, 1 \mathrm{H}), 5.36$ (s, 2H), 2.52 (s, 3H); ${ }^{13} \mathrm{C}$ NMR (125 MHz, DMSO-d 6 ) $\delta 197.26,145.42,142.23,139.96$, $139.74,136.85,136.32,135.84,133.86,131.96,130.17,129.34,128.74,128.36,127.85,127.65,127.35$, $127.14,127.03,125.02,124.37,123.98,122.05,121.45,120.49,119.63,118.94,118.02,110.42,109.63$, 43.41, 39.38, 26.75.LC-MS m/z: $576[\mathrm{M}+\mathrm{H}]+$ Elemental analysis, Calculated, \%: $\mathrm{C}_{31} \mathrm{H}_{23} \mathrm{BrN}_{6} \mathrm{O}: \mathrm{C}, 64.70 ; \mathrm{H}$, 4.03; N, 14.60; Found \%:C, 64.61; H, 3.94; N,14.51; 
1-(4-(4-((2-(6-bromopyridin-2-yl)-4,5-diphenyl-1 Himidazol-1-yl)methyl)-1 H-1,2,3-triazol-1-yl)phenyl)ethan-1one(7k)Yield 78\%, mp: $149-151^{\circ} \mathrm{C}$; Rf $=0.35$ (EtOAc:n-Hexane 2:3); N,14.51; ${ }^{1} \mathrm{H}$ NMR (500 MHz, DMSOd6) $8.42(\mathrm{~s}, 1 \mathrm{H}), 7.92(\mathrm{~d}, \mathrm{~J}=7.85 \mathrm{~Hz}, 1 \mathrm{H}), 7.80(\mathrm{~d}, \mathrm{~J}=8.43 \mathrm{~Hz}, 2 \mathrm{H}), 7.74(\mathrm{~d}, \mathrm{~J}=8.43 \mathrm{~Hz}, 4 \mathrm{H}), 7.63$ (dd, J = 7.85, 7.47 Hz, 1H), 7.59 (d, J = 7.85 Hz, 2H), 7.43 (dd, J = 7.85 7.45 Hz, 2H), 7.43 (dd, J = 7.85, 7.45 Hz, 1H), $7.41(\mathrm{~d}, \mathrm{~J}=7.85 \mathrm{~Hz}, 1 \mathrm{H}), 7.40(\mathrm{~m}, \mathrm{~J}=7.457 .85 \mathrm{~Hz}, 3 \mathrm{H}), 7.34(\mathrm{t}, \mathrm{J}=7.45 \mathrm{~Hz}, 1 \mathrm{H}), 5.35(\mathrm{~s}, 2 \mathrm{H}), 2.56(\mathrm{~s}$, $3 \mathrm{H}) ;{ }^{13} \mathrm{C}$ NMR (125 MHz, DMSO-d 6 ) $\delta 196.84,145.47,142.16,139.98,139.74,137.85,136.22,135.84$, 133.84, 131.96, 130.32, 130.17, 128.74, 128.36, 127.85, 127.63, 127.12, 127.03, 125.02, 124.39, 123.95, 122.07, 119.53, 118.97, 118.85, 110.47, 109.63, 43.42, 39.30, 26.40.LC-MS m/z: 576 [M+H]+ Elemental analysis, Calculated, \%: $\mathrm{C}_{31} \mathrm{H}_{23} \mathrm{BrN}_{6} \mathrm{O}: \mathrm{C}, 64.70 ; \mathrm{H}, 4.03 ; \mathrm{N}, 14.60$; Found \%:C, 64.61; $\mathrm{H}, 3.94$;

\section{2-bromo-6-(1-((1-(4-nitrophenyl)-1 H1,2,3-triazol-4-yl)methyl)-4,5-diphenyl-1 H-imidazol-2-}

yl)pyridine(7l)Yield 80\%, mp: 160-162 ${ }^{\circ} \mathrm{C}$; Rf = 0.34 (EtOAc:n-Hexane 2:3); ${ }^{1} \mathrm{H}$ NMR $(500 \mathrm{MHz}, \mathrm{DMSO}-d 6) \delta$ $8.49(\mathrm{~s}, 1 \mathrm{H}), 8.34(\mathrm{~d}, \mathrm{~J}=9.20 \mathrm{~Hz}, 2 \mathrm{H}), 7.92(\mathrm{~d}, \mathrm{~J}=7.85 \mathrm{~Hz}, 1 \mathrm{H}), 7.79(\mathrm{~d}, \mathrm{~J}=9.20 \mathrm{~Hz}, 2 \mathrm{H}), 7.74(\mathrm{~d}, \mathrm{~J}=7.85$ $\mathrm{Hz}, 2 \mathrm{H}), 7.63$ (dd, J = $7.857 .82 \mathrm{~Hz}, 1 \mathrm{H}), 7.59$ (d, J = 7.85 Hz, 2H), 7.43 (dd, J = 7.85, $7.45 \mathrm{~Hz}, 2 \mathrm{H}), 7.41$ (d, $\mathrm{J}=7.85 \mathrm{~Hz}, 1 \mathrm{H}), 7.40(\mathrm{~m}, 3 \mathrm{H}), 7.35(\mathrm{t}, \mathrm{J}=7.45 \mathrm{~Hz}, 1 \mathrm{H}), 5.34(\mathrm{~s}, 2 \mathrm{H}) ;{ }^{13} \mathrm{C}$ NMR $\left(125 \mathrm{MHz}, \mathrm{DMSO}-\mathrm{d}_{6}\right) \delta$ $146.05,145.47,142.17,139.98,139.75,135.82,133.86,131.97,130.17,128.76,128.36,127.84,127.66$, $127.13,127.04,126.17,125.03,124.38,123.98,121.95,120.14,119.53,118.92,118.87,110.42,109.63$, 43.48, 39.31.LC-MS m/z: 579 [M+H]+ Elemental analysis, Calculated, $\%: \mathrm{C}_{29} \mathrm{H}_{20} \mathrm{BrN}_{7} \mathrm{O}_{2}$ Exact Mass: $\mathrm{C}$, $60.22 ; \mathrm{H}, 3.49 ; \mathrm{N}, 16.95$; Found \%:C, C, 60.16; H, 3.39; N, 16.89;

\section{Declarations}

\section{Acknowledgements}

All the authors are thankful to the Head, Department of chemistry, Osmania University, Hyderabad for providing laboratory facilities. Lakavath Ramdas is thankful to CSIR for financial assistance in the form of fellowship and one of the authors We thank Central Facilities and Research Development (CFRD) analytical team for providing spectral analytical facilities.

\section{Conflict of interest}

Authors declare no conflict of interest

\section{References}

1. Jemal, F. Bray, M.M. Center, J. Ferlay, E. Ward, D. Forman, Global cancer statistics, CA: a cancer journal for clinicians, 61 (2011) 69-90.

2. GLOBOCAN 2020.

3. Akhtar, A.A. Khan, Z. Ali, R. Haider, Y. Shahar, Structure-activity relationship (SAR) study and design strategies of nitrogen-containing heterocyclic moieties for their anticancer activities, Eur. J. Med. Chem. 125 (2017) 143-189. 
4. Kamal, A.V.S. Rao, M.V.P.S. Vishnuvardhan, T.S. Reddy, K. Swapna, C. Bagul, N.V.S. Reddy, V. Srinivasulu, Synthesis of 2-anilinopyridyl-triazole conjugates as antimitotic agents, Org. Biomol. Chem. 13 (2015) 4879-4895.

5. Prasad, V.L. Nayak, P.S. Srikanth, M.F. Baig, N.V.S. Reddy, K.S. Babu, A. Kamal, Synthesis and biological evaluation of 1-benzyl-N-(2-(phenyl-amino) pyridin-3-yl)-1H-1,2,3-triazole-4-carboxamides as antimitotic agents, Bioorg. Chem. 83 (2019) 535-548.

6. Bai, C. Liao, Y. Liu, X. Qin, J. Chen, Y. Qiu, D. Qin, Z. Li, Z.C. Tu, S. Jiang, Structure-based design of potent nicotinamide phosphoribosyltransferase inhibitors with promising in vitro and in vivo antitumor activities, J. Med. Chem. 59 (2016) 5766-5779.

7. Dong, W. Chen, X. Wang, X. Yang, Y. Xu, P. Wang, W. Zhang, Y. Rao, C. Miao, C. Sheng, Small molecule inhibitors simultaneously targeting cancer metabolism and epigenetics: discovery of novel nicotinamide phosphoribosyltransferase (NAMPT) and histone deacetylase (HDAC) dual inhibitors, J. Med. Chem. 60 (2017) 7965-7983.

8. Travelli, S. Aprile, R. Rahimian, A.A. Grolla, F. Rogati, M. Bertolotti, F. Malagnino, R. Paola, D. Impellizzeri, R. Fusco, V. Mercalli, A. Massarotti, G. Stortini, S. Terrazzino, E.D. Grosso, G. Fakhfouri, M.P. Troiani, M.A. Alisi, G. Grosa, G. Sorba, P.L. Canonico, G. Orsomando, S. Cuzzocrea, A.A. Genazzani, U. Galli, G.C. Tron, Identification of novel triazole-based nicotinamide phosphoribosyltransferase (NAMPT) inhibitors endowed with anti-proliferative and antiinflammatory activity, J. Med. Chem. 60 (2017) 1768-1792.

9. Theeramunkong, U. Galli, A.A. Grolla, A. Caldarelli, C. Travelli, A. Massarotti, M.P. Troiani, M.A. Alisi, G. Orsomando, A.A. Genazzani, G.C. Tron, Identification of a novel NAMPT inhibitor by combinatorial click chemistry and chemical refinement, MedChemComm 6 (2015) 1891-1897.

\section{Figures}

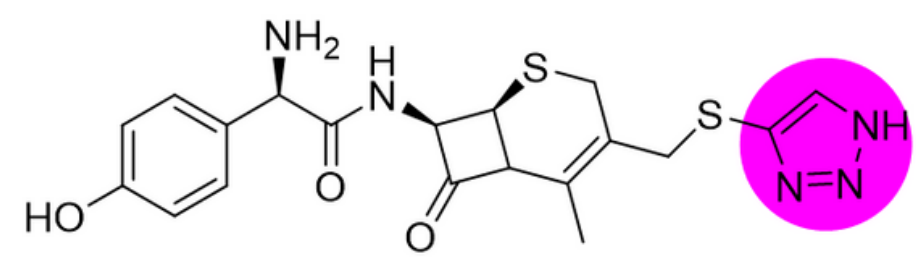

Cefatrizine[a]

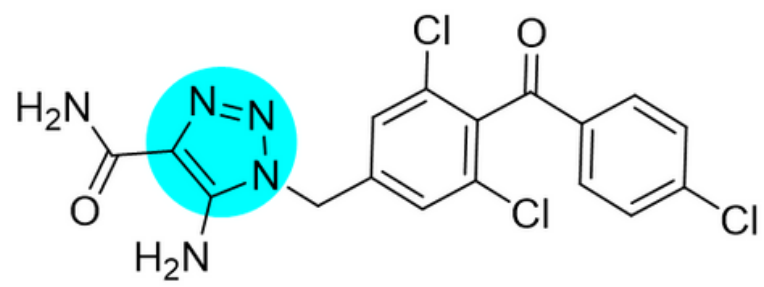

Carboxyamidotriazole[b]

\section{Figure 1}

(a), (b) The chemical structures of anticancer agents containing 1,2,3 triazole as a scaffold. 


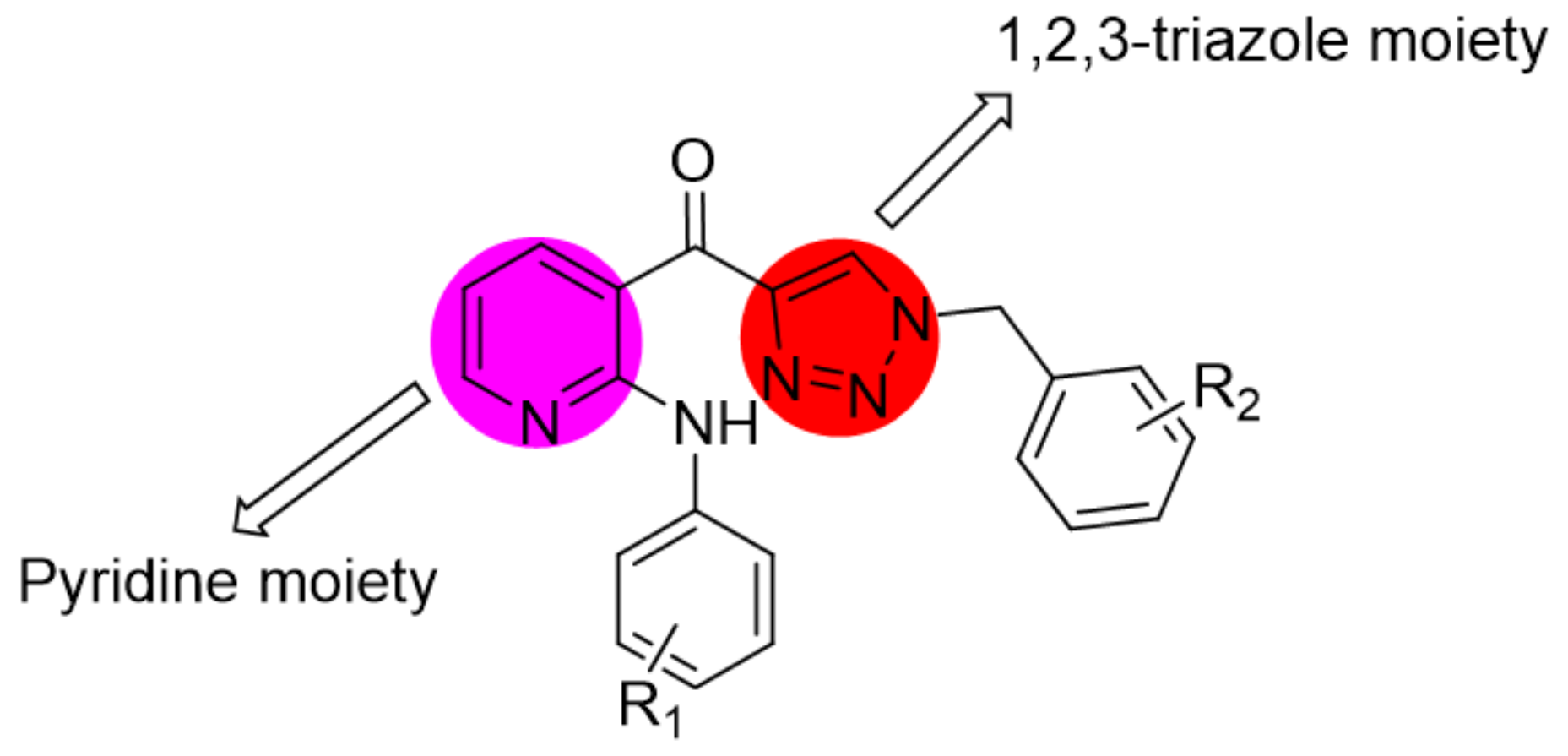

Figure 2

The chemical structures of anticancer agents containing 1,2,3 triazole pyridine hybrid scaffold.

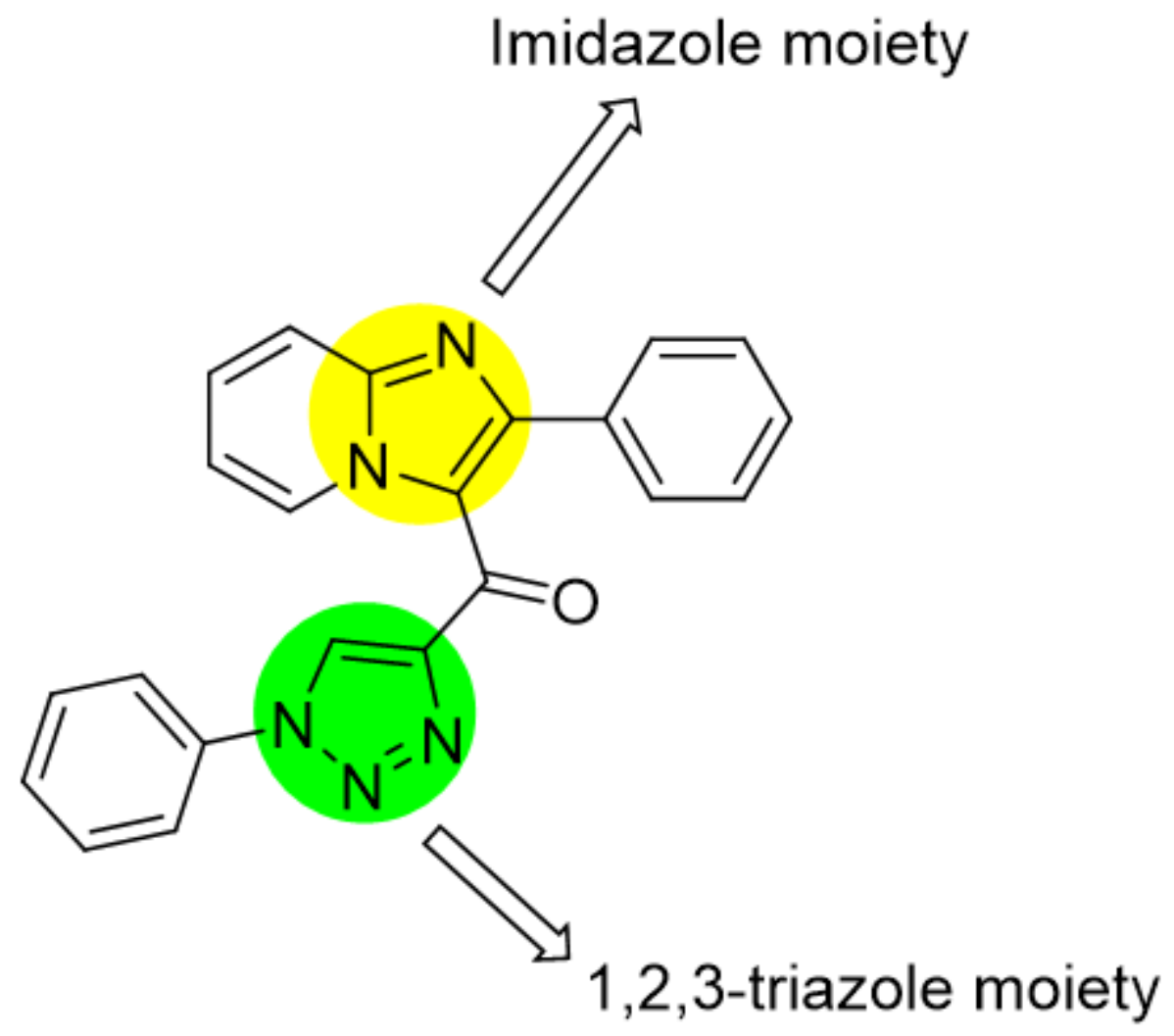

Figure 3 
The chemical structures of anticancer agents containing 1,2,3 triazole pyridine hybrid scaffold.

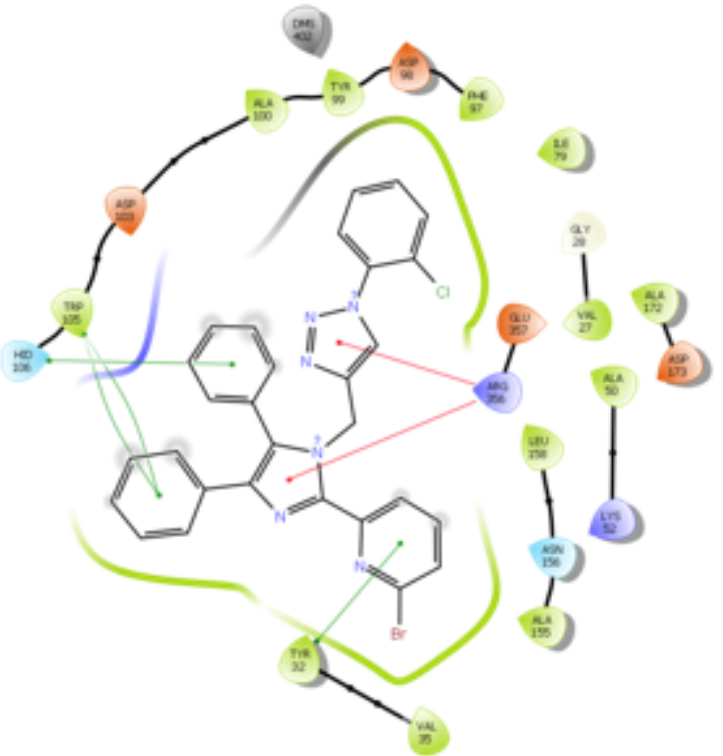

$7 \mathrm{~b}$

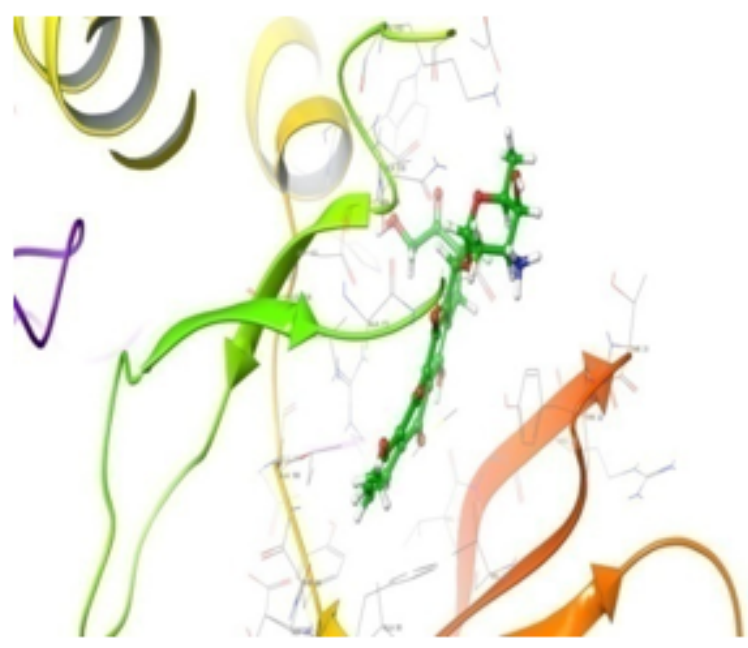

$7 \mathrm{~b}$

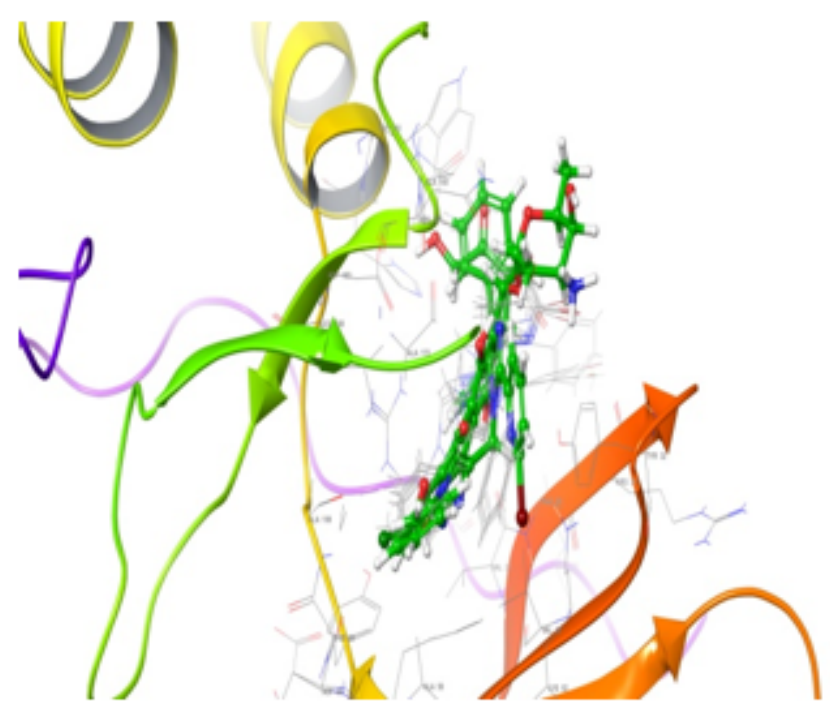

Figure 4

Dock poses and ligand interaction diagrams of molecules $7 \mathrm{~b}$.

\section{Supplementary Files}

This is a list of supplementary files associated with this preprint. Click to download.

- scheme1.png

- Supportinginformation.docx 